\title{
Gradhiva
}

Revue d'anthropologie et d'histoire des arts

Wampum : les perles de la diplomatie

\section{Les wampums au Québec du XIX siècle à aujourd'hui Appropriation, disparition, identification}

The wampum in Quebec from the 19th century until today. Appropriation, disappearance, identification

Jonathan Lainey

\section{CpenEdition}

Journals

Édition électronique

URL : https://journals.openedition.org/gradhiva/6059

DOI : 10.4000/gradhiva.6059

ISSN : 1760-849X

Traduction(s) :

Wampum in Quebec from the 19th Century to the Present Day: Appropriation, Loss, Identification -

URL : https://journals.openedition.org/gradhiva/6238 [en]

\section{Éditeur}

Musée du quai Branly Jacques Chirac

\section{Édition imprimée}

Date de publication : 2 février 2022

Pagination : 98-117

ISBN : 978-2-35744-133-0

ISSN : 0764-8928

Référence électronique

Jonathan Lainey, «Les wampums au Québec du xIx $x^{\text {e }}$ siècle à aujourd'hui Appropriation, disparition, identification », Gradhiva [En ligne], 33 | 2022, mis en ligne le 02 février 2022, consulté le 15 septembre 2022. URL : http://journals.openedition.org/gradhiva/6059; DOI : https://doi.org/10.4000/gradhiva. 6059 


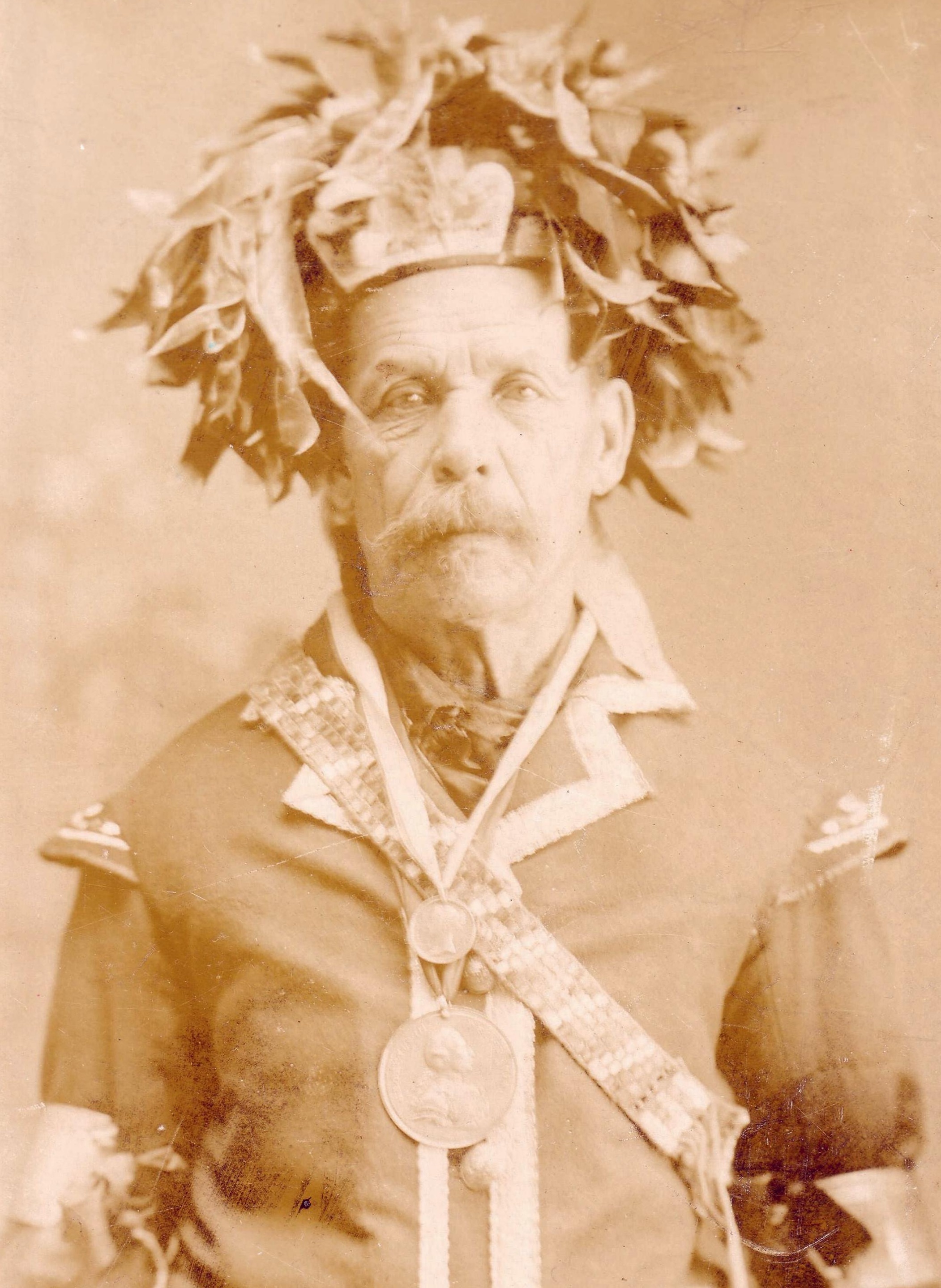


Les wampums

au Québec

du XIX ${ }^{\mathrm{e}}$ siècle

à aujourd'hui

Appropriation,

disparition,

identification

Mots clefs:
Québec, Hurons-Wendat,

collecte ethnographique,

Kanien'kehá:ka, musée McCord

Jonathan Lainey 
Après plus de deux siècles de circulation régulière dans des contextes diplomatiques impliquant les nations autochtones et européennes du nord-est de l'Amérique, les colliers de wampum virent leur utilisation réduite au xIXe siècle. Depuis l'affaiblissement de leur rôle politique jusqu'à leur intégration dans des collections privées ou muséologiques, I'histoire de ces objets prit différentes formes et suivit plusieurs directions. La présentation de cas particuliers de wampums ayant été ou étant toujours conservés sur le territoire actuel du Québec est utile pour expliquer en partie pourquoi ces objets de nature collective ont pu sortir de leur communauté d'origine pour se retrouver dans des musées. Ces exemples servent aussi à illustrer la manière dont une signification ou fonction nouvelles ont été imposées aux wampums par les collectionneurs privés non autochtones au détriment du sens initial que leur attribuaient leurs propriétaires d'origine.

\section{Cet article reprend et bonifie certains éléments d'une série de publications parues entre 2004 et aujourd'hui.}

2. Ces perles étaient acquises par les Hollandais et les Anglais auprès des peuples autochtones producteurs en échange de différents objets de traite manufacturés en Europe. Avec ces perles, les Européens se tournaient ensuite vers les nations situées plus à l'ouest, qui elles leur fournissaient les fourrures. En expédiant les perles des côtes vers l'intérieur, on favorisait l'exportation des fourrures vers les colonies, où les investisseurs faisaient de larges profits en les envoyant en Europe.

3. Pour des exemples de la persistance des usages des wampums jusqu'au $x x^{e}$ siècle voir Lainey 2004: 82-86.

\section{INTRODUCTION 1}

Fabriquées à partir de coquillages marins des côtes atlantiques, les perles dont sont faits les wampums étaient un bien d'échange important dans la traite des fourrures, en pleine expansion au début du XVII ${ }^{\mathrm{e}}$ siècle en Amérique du Nord-Est. C'est à partir de cette denrée que les Hollandais et les Anglais purent avoir accès aux milliers de fourrures nécessaires au commerce ${ }^{2}$. Les peuples de langue et de culture iroquoiennes de l'intérieur des terres les utilisaient dans leurs relations diplomatiques avec les nations voisines. Les perles, alors tissées en colliers de diverses tailles, étaient offertes pour accompagner les discours, les rendre légitimes et officiels. Un mémoire français rédigé au début du XVIII ${ }^{\mathrm{e}}$ siècle rend compte de l'importance de ces objets lors des rencontres officielles en Amérique:

\begin{abstract}
Ces coliers sont si nécessaires à ceux qui parlent d'affaires, au nom des nations, qu'on n'ajouteroit aucune foy à leurs paroles si préalablement ils ne présentoient à celuy avec lequel ils ont à traiter un colier qu'ils étendent devant luy. Après le discours fini, le sauvage auquel on s'adresse prend le colier et en remet un autre à la place pour faire sa réponse. (BnF vers 1725)

[voir texte intégral dans ce numéro, section Archives]
\end{abstract}

Certains wampums étaient conservés sur de longues périodes pour que les messages qu'ils portaient demeurent et qu'on se souvienne des termes des ententes passées et des engagements mutuels. L'échange de wampums obéissait aux rèǵles protocolaires de nombreuses nations du nord-est de l'Amé- rique, également adoptées par les Européens dans leurs transactions diplomatiques pendant les deux siècles qui suivirent le contact entre l'Europe et l'Amérique (XVII ${ }^{\mathrm{e}}$ et XVIII ${ }^{\mathrm{e}}$ siècles).

On affirme généralement que les wampums diplomatiques cessèrent de circuler régulièrement après la guerre de 1812-1814, dernier conflit colonial opposant la Couronne britannique aux Américains ainsi que leurs alliés autochtones respectifs. Certains auteurs cherchent à identifier la date de la fin de l'utilisation des wampums en diplomatie. Il serait imprudent voire présomptueux de vouloir définir une date précise. Si les échanges de colliers entre les nations autochtones et européennes ont cessé à un moment donné, ce n'est pas nécessairement le cas au sein des villages et communautés autochtones eux-mêmes ou encore à l'intérieur d'une même nation. Des pratiques locales peuvent avoir perduré sans que cela ait laissé de traces dans les archives que nous consultons aujourd'hui. Même si les wampums ne circulaient plus comme au XVIII ${ }^{\mathrm{e}}$ siècle et que leurs usages avaient décliné ou s'étaient substantiellement modifiés avec le temps, plusieurs nations ont conservé longtemps leurs wampums auxquels elles se référaient pour rappeler certains principes fondateurs et événements historiques, ou encore pour ponctuer ou officialiser certaines cérémonies. Ainsi les wampums continuent d'être mentionnés, présentés, utilisés lors d'événements publics ou de rencontres formelles sans pour autant être offerts ou échangés ${ }^{3}$.

Malgré tout, il demeure évident que la majorité des wampums se sont retrouvés d'une manière ou d'une autre dans les collections privées ou dans les musées. L'histoire de ces objets au cours des cent cinquante dernières années - depuis l'affaiblissement de leur rôle politique jusqu'à leur intégration dans des collections privées ou muséologiques - a pris différentes 


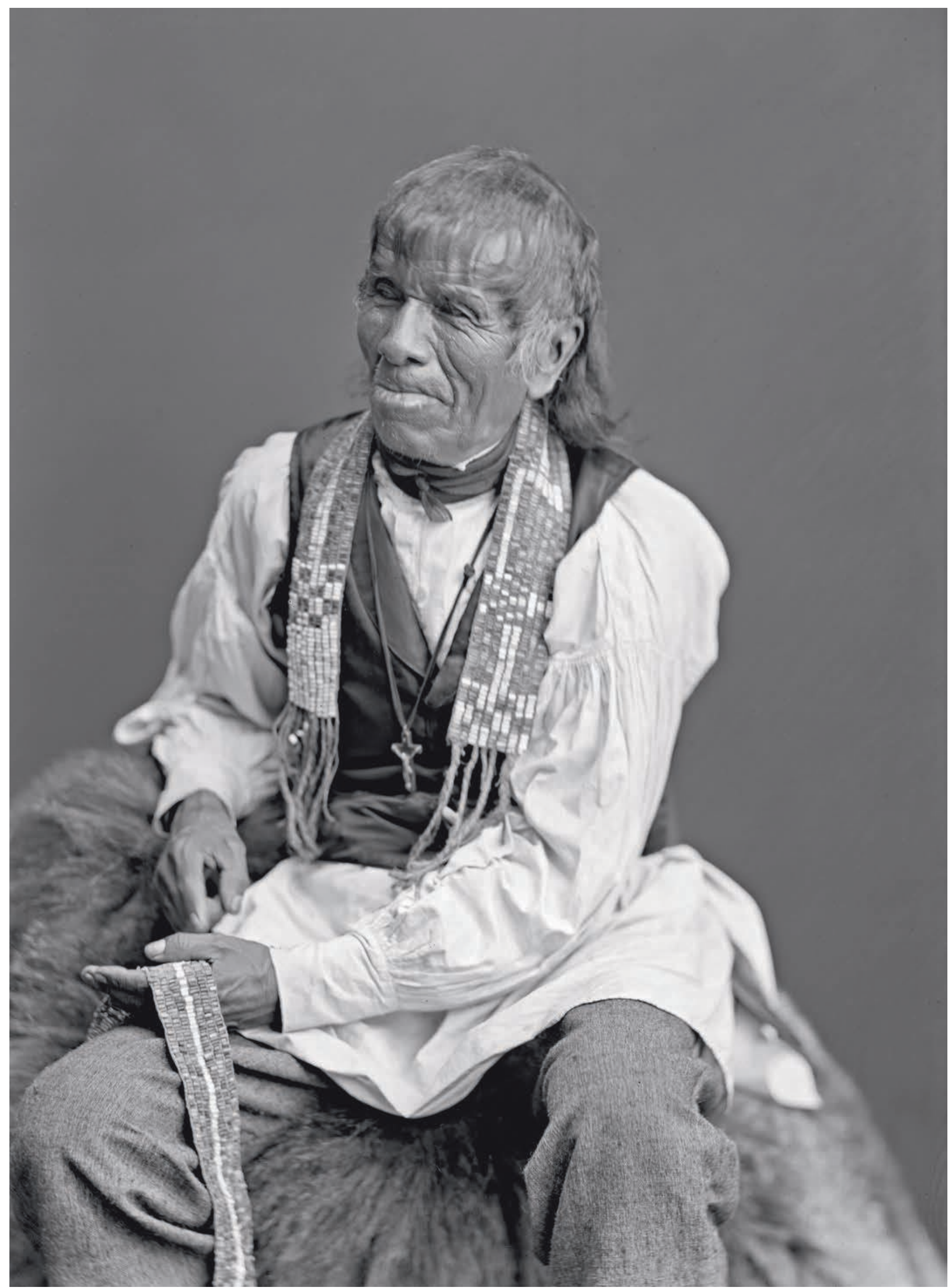

fig. 1. William Notman

Le chefonondaga Isaac Hill Kaweneseronton de la Confédération des Six Nations, un wampum à la main et un autre autour du cou, Montréal, 1870. Tirage sur papier albuminé, 13.7 × $10 \mathrm{~cm}$. Montréal, musée McCord, I-48873.1. 
4. Sur le passage

du patrimoine matériel et documentaire de la sphère communautaire à la sphère privée chez les Wendat, voir: Lainey 2004:142-162 et $183-185$. formes et suivi plusieurs directions. On sait que certains wampums ont été légués ou vendus afin de bénéficier de meilleures conditions de conservation, particulièrement aux États-Unis. Craignnant leur dispersion éventuelle ou leur lente destruction, certains chefs ont placé leurs wampums dans des universités ou musées nationaux qu'ils ont officiellement nommés les «gardiens des wampums» (Lainey 2004: 143-144).

Au Québec, les wampums ne semblent pas avoir quitté leur communauté pour les mêmes raisons. Plusieurs ont fini dans des collections privées qu'on retrouve souvent dans les collections muséologiques contemporaines. Un des motifs expliquant la vente de ces objets serait le changement du mode de propriété qui est passé progressivement, entre la fin du XIX ${ }^{\mathrm{e}}$ et le début du $\mathrm{Xx}^{\mathrm{e}}$ siècle, du domaine collectif et national à la sphère individuelle et familiale.

Afin d'illustrer ces réalités historiques, nous allons nous pencher sur des exemples particuliers de wampums ayant été ou étant toujours conservés sur le territoire actuel du Québec et qui sont relativement bien documentés. Ces wampums, dont certains font partie de la collection du musée McCord, à Montréal, alors que d'autres étaient conservés au sein d'institutions reliǵieuses, concernent surtout les Wendat de Wendake (autrefois nommés les Hurons de Lorette), mais aussi les Kanien'kehá:ka de Kahnawake et de Kanesatake (anciens Mohawks du Sault-Saint-Louis et du Lac-des-Deux-Montagnes), le premier village étant situé près de la ville de Québec, les deux autres de Montréal. Ces études de cas serviront à expliquer en partie pourquoi ces objets de nature collective ont pu sortir de leur communauté d'origine pour se retrouver dans des collections muséologiques. Sans être parfaitement représentatifs de ce qu'il est advenu de l'ensemble des wampums aux XIX ${ }^{\mathrm{e}}$ et $\mathrm{XX}^{\mathrm{e}}$ siècles, les exemples fournis offrent cependant un aperçu de certaines réalités touchant à la culture matérielle des nations autochtones du nord-est de l'Amérique.

L'histoire de ces objets servira du même coup à illustrer la manière dont, lors de la collecte ethnologique, une signification ou fonction nouvelles ont été imposées aux wampums par les non-autochtones au détriment du sens initial que leur attribuaient leurs propriétaires d'oriǵine. En effet, une fois entre les mains de collectionneurs privés, la siǵnification première de ces objets fut parfois complètement transformée, laissant place aux idées et fantasmes de ces nouveaux propriétaires. Qu'elle soit matérielle ou immatérielle, cette réappropriation n'est pas sans conséquences sur la perception que nous avons aujourd'hui de ces objets.

\section{CHANGEMENTS SOGIOPOLITIQUES ET CHANGEMENT DE MODE DE PROPRIÉTÉ}

Chez les Wendat de Wendake, les wampums étaient conservés par les chefs, lesquels formaient un conseil pouvant compter jusqu'à dix individus au début du XIX siècle. Ces chefs étaient nommés à vie par leur clan et leur famille, de sorte que certains ont occupé cette position pendant plusieurs dizaines d'années. Lorsque l'un d'eux décédait, un nouveau chef était nommé pour le remplacer. L'ensemble de ces porte-parole et représentants claniques et familiaux veillaient au maintien et à la transmission des connaissances et du savoir collectifs et conservaient les documents écrits ainsi que les objets en lien avec l'histoire nationale. Les wampums en faisaient partie, confiés à la garde des chefs qui en étaient les dépositaires, et non les propriétaires.

Lorsque à partir de la fin du XIX ${ }^{\mathrm{e}}$ siècle le système de représentation traditionnel a commencé à s'affaiblir, se perpétuant difficilement, les wampums ont progressivement quitté le cercle des chefs à vie pour être transmis aux membres de leurs familles. Ce phénomène se produisit lorsque les derniers chefs traditionnels élus à vie ont été substitués par d'autres, élus selon le système triennal imposé par la loi sur les Indiens, une loi fédérale raciste promulguée en 1876 qui contrôle l'essentiel de la vie légale et civique des «Indiens », du berceau au cercueil, dans le but de les assimiler à la société dominante. Ce passage de la sphère collective à la sphère privée ressort clairement des testaments rédigés par les chefs wendat de l'époque: ils lèguent les objets historiques tels que les wampums et les médailles à leurs descendants, et non au chef subséquent ${ }^{4}$. Qu'ils soient restés dans le giron de la famille n'est pas sans lien avec le fait que les titres de chefs étaient héréditaires et transmis au sein des clans: que les wampums ne quittent pas la famille du défunt respecte donc une certaine logique coutumière. Concrètement, cette pratique signifie qu'un enfant devenait détenteur d'un wampum même s'il n'occupait pas le poste de chef traditionnel. Le prêtre wendat Prosper Vincent (1842-1915) explique à l'ethnologue Marius Barbeau, en 1911, ce phénomène qu'il a lui-même observé:

Quandj'étais jeune, j'ai eu connaissance
que le grand chef gardait à sa main un
bon nombre de colliers de porcelaine [...].
Ces colliers-là dans ce temps-là étaient
considérés comme la propriété de la tribu.
Plus tard cette propriété est devenue
divisible, ce qui a conduit à la vente
de la plupart de ces colliers.
(Cité dans Lainey 2004: 147)

Vincent n'a pas tort lorsqu'il souligne l'effet non néǵligeable que ce changement de mode de propriété a eu sur la vie des wampums: la possibilité de les vendre. Désormais considérés par certains individus comme des objets personnels, ceux-ci crurent avoir le droit de s'en départir. L'anthropologue Horatio Hale a constaté le même phénomène chez les Wyandots d'Anderdon (Michigan, États-Unis) à la toute fin du XIX siècle, au moment où il acquit les quatre wampums aujourd'hui exposés au Pitt Rivers Museum d'Oxford:

\section{Le chef dit posséder des colliers qui étaient sa propriété privée et qu'il pouvait me vendre. J'en déduisis qu'il s'agissait de}


colliers dont ils ne se servaient plus et que les anciens gardiens des wampums, conformément à l'usage de la communauté, avaient laissés à sa disposition. (Hale 1897: 233, traduction de l'auteur)

Si ces changements sociopolitiques expliquent en partie pourquoi certains ont estimé pouvoir vendre des objets diplomatiques de propriété collective à des collectionneurs privés, cela ne signifie pas qu'ils avaient le droit de le faire. Il est évident que ces particuliers ont vendu des objets ayant une signification et une fonction qui dépassaient de loin leur propre personne et concernaient toute la nation. D'ailleurs, on sait qu'à Wendake, le conseil des chefs a parfois tenu à récupérer ou à protéger ce patrimoine matériel et documentaire passé entre les mains d'individus ne représentant pas la nation. En 1923, par exemple, le grand chef Ovide Sioui s'est plaint auprès du ministère des Affaires indiennes que Pierre-Albert Picard, le petit-fils de François-Xavier Picard, un ancien chef traditionnel élu à vie, "dét[enait] en sa possession des documents précieux et autres effets qui sont la propriété de la tribu» (BAC 1923-1925). En 1898, le tribunal de New York dut se pencher sur la question de la légalité des transferts de propriété des wampums qui avaient déjà eu lieu. Les Onondaǵas, membres de la confédération Haudenausonee, plaidèrent qu'un individu ne pouvait les vendre ou les donner puisqu'il s'agissait d'objets appartenant à la confédération. En retour, les avocats de la partie adverse arguèrent que la confédération ayant cessé d'exister en tant que telle depuis longtemps, les objets étaient devenus des objets de famille légués en héritaǵe et des «reliques» de propriété individuelle (The Gazette 1898: 6).

Parfois, les chefs ou autres membres de la nation ne se sont rendu compte de la disparition des wampums que des années après leur vente. Les démarches qu'ils ont alors intentées pour les retrouver sont ardues et s'avèrent le plus souvent infructueuses, notamment parce que les traces documentaires laissées par les différents collectionneurs contribuent à brouiller les pistes, modifiant, transformant et omettant des informations essentielles à l'histoire de ces objets (Bruchac 2018).

\section{CHANGEMENTS DE SIGNIFICATION ET D'INTERPRÉTATION}

Une fois le wampum sorti de la communauté, la signification et la provenance qu'on lui attribue sont entièrement dépendantes des dires et de la volonté de ceux qui le possèdent, au détriment de leur oriǵine historique précise. En effet, il acquiert parfois un sens qui travestit complètement le message originel dont il était porteur à l'époque où il était échangé dans un contexte diplomatique international, et ayant vocation à être remémoré au fil du temps afin de préserver les termes de l'entente initiale. La transformation de la signification de ces wampums à travers les époques ne relève donc pas d'un phénomène social et culturel qui verrait l'histoire orale propre à une culture évoluer et changer de sens, mais bien de la négation, de l'usurpation et de l'altération des récits de l'Autre par l'appropriation de la culture matérielle qui en témoigne. Pour illustrer cette réalité historique, prenons les exemples concrets de deux wampums importants, actuellement conservés au musée McCord à Montréal, dont l'histoire est particulièrement bien documentée.

\section{LE «GRAND GOLLIER DE GUERRE »DES WENDAT}

Conservé au musée McCord, ce wampum très connu est un symbole de l'alliance militaire entre d'une part la Couronne britannique et d'autre part la Nation huronne-wendat ainsi que probablement les Sept-Nations du Canada, une confédération autochtone regroupant les nations établies le lonǵ de la vallée du Saint-Laurent, dont les Wendat. Le motif central figurant sur ce wampum est la hache donnée par George III dont les Hurons-Wendat acceptèrent de se servir afin de défendre les intérêts de leur nouvel allié dans le contexte de la guerre de Conquête ${ }^{5}$. Il aurait été offert à la fin du régime français en Amérique du Nord, lorsque les Britanniques se sont alliés aux nations autochtones, réclamant leur neutralité ou leur soutien pour conquérir les territoires français, en échange de la protection de leurs coutumes et de leurs territoires. C'est dans ce contexte politique particulier que certains des premiers traités de paix et d'amitié ont été conclus entre les Britanniques et les Premières Nations vivant le long du fleuve Saint-Laurent ${ }^{6}$.

Lorsque quatre leaders wendat se rendirent à Londres en 1824-1825 pour y rencontrer le roi George IV dans le but de faire valoir leurs droits fonciers sur le territoire de la seigneurie de Sillery, dans la réǵion de Québec, ils apportèrent ce wampum afin de rappeler à la Couronne cette ancienne alliance qui les unissait. Une gravure a immortalisé le grand chef Nicolas Vincent Tsawenhohi tenant à la main ledit wampum (fig. 2). Le texte figurant au bas de la gravure indique on ne peut plus clairement la symbolique de la hache représentée sur le wampum: «Le chef tient dans sa main le wampum ou le collier sur lequel est représenté le tomahawk offert par feu Sa Majesté, George III » (traduction de l'auteur).

Le cheminement de ce wampum fondamental dans l'histoire des Wendat est particulièrement représentatif du destin de plusieurs autres colliers de wampum à la même époque. Après être passé entre les mains des chefs qui se sont succédé pendant plus d'un siècle, ce wampum est demeuré au sein de la famille de l'un des derniers chefs élus à vie selon la coutume wendat. En 1883, au moment du décès du grand chef héréditaire François-Xavier Picard Tahourenché, le wampum est resté dans la famille Picard, et c'est Paul, son fils, qui en eut la garde, l'ayant reçu en héritage ainsi que d'autres objets historiques importants. Paul ne put le transmettre à ses propres enfants puisqu'il perdit le précieux wampum, cédé au riche notaire et collectionneur
5. Sur ce wampum et sa représentation à travers le temps, voir: Lainey et Whitelaw 2021.

6. Il est aussi possible que les Wendat aient reçu et accepté la hache de George III dans le contexte du conflit armé qui opposa la Couronne britannique aux rebelles américains au milieu des années 1770. 


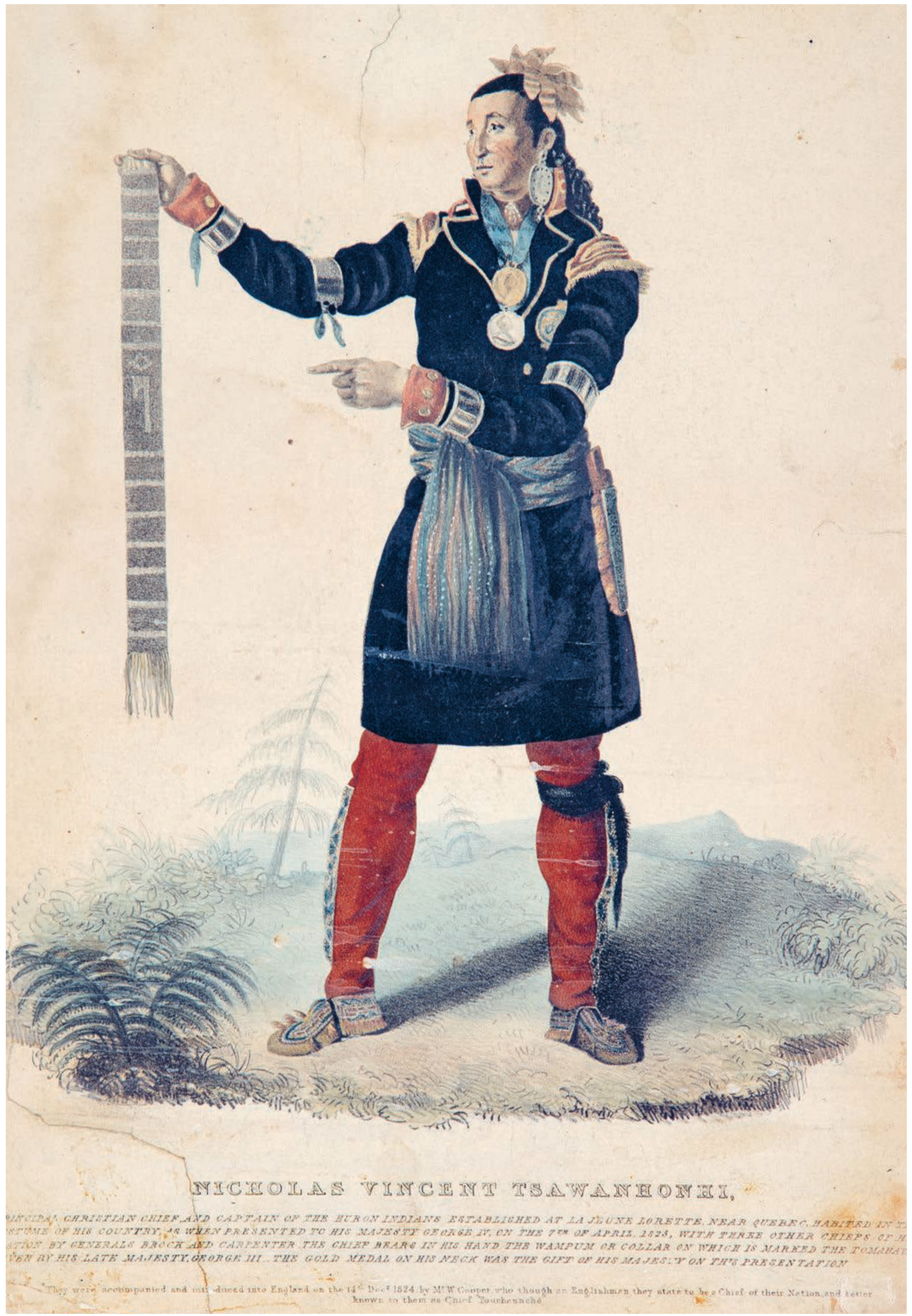

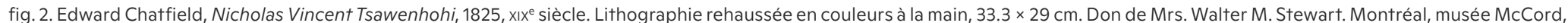

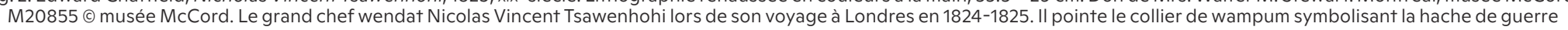
de George III et arbore des médailles de George III et IV. 
de la ville de Québec, Cyrille Tessier (1835-1931), alors qu'il devait faire face à d'importantes difficultés financières liées notamment au procès qui l'opposa à ses sœurs, justement à propos de questions successorales (Lainey 2004: 121-126 et 149).

Écarté de la vue du public, ce wampum est demeuré jalousement conservé dans la collection de la famille Tessier, jusqu'à sa vente au musée McCord en 1957. À partir de ce moment-là, le sens originel du wampum qui renvoyait à une alliance militaire ayant permis l'établissement des Britanniques en Amérique du Nord-Est a été complètement remis en question avant d'être simplement iǵnoré. En 1951, lorsque Joachim Des Rivières Tessier, le fils de Cyrille le collectionneur, prêta le wampum de sa collection pour une exposition commémorant la fondation de Détroit par Antoine de La Mothe-Cadillac en 1701, il lui donna un sens qui n'avait rien à voir avec son message initial: on dit alors qu'il était un des trois wampums échangés lors de la la Grande Paix de Montréal de 1701 impliquant les Français, et que son symbole principal était plutôt la hache de guerre enterrée pour l'occasion (Richardson 1951: 49 et 51). D’un wampum échangé au moment où étaient établis les fondements d'une nouvelle relation autochtone-britannique qui allait garantir le succès de l'implantation de la Couronne anǵlaise en Amérique, celui-ci est devenu un objet rappelant faussement une paix générale qui impliquait les Français quelque soixante ans plus tôt. On est donc passé d'une alliance militaire avec les Britanniques à une paix générale avec les Français. Le renversement de sens est complet. Même si l'imaǵe publiée en 1825 était là pour rappeler constamment aux historiens et au public que ce collier représentait bel et bien la hache de George III, les nouveaux propriétaires jugèrent qu'il leur était possible de contredire et de taire le véritable message du wampum. Et c'est cette même interprétation erronée qui accompaǵna le wampum vendu au McCord en 1957 lorsqu'il franchit les portes du musée, et qui fut reprise par la suite dans de nombreuses et diverses publications par des chercheurs chevronnés n'ayant pas pris soin de la questionner et d'en vérifier les sources (Lainey et Whitelaw 2021: 192-194).

La transformation du sens véritable des wampums n'est pas anodine et sans conséquence. Ce wampum a été échangé lors de la conclusion d'une entente fondatrice et est issu d'une relation historique qui lie les nations autochtones à la Couronne britannique, et vice-versa. Le geste extraordinaire des leaders wendat qui, exceptionnellement, réussirent à s'entretenir personnellement avec le roi George IV en 1825 afin de lui rappeler les devoirs et responsabilités définissant la relation sincère entre alliés, a été ignoré. Les promesses mutuelles faites lors de la Conquête britannique et qui auraient dû répondre aux intérêts des Wendat ont ainsi été négligées jusqu'à en être oubliées. La relégation et le déni du contenu de ces ententes contribuent à réduire le poids de l'affirmation de droits ancestraux autochtones ayant potentiellement, aujourd'hui encore, une valeur légale.

\section{LE «WAMPUM AUX DEUX CHIENS » DE KANESATAKE}

7. Sur ces événements des années 1780, voir: Lainey 2013: 99-108.

Le même phénomène d'appropriation et de transformation de sens s'est produit à Kanesatake, où un wampum tout aussi important que le précédent fut vendu par un particulier au début du $\mathrm{xx}^{\mathrm{e}}$ siècle. Généralement connu sous le nom de Troo-Dog Wampum Belt (TDWB), ce «wampum aux deux chiens », aujourd'hui conservé au musée McCord, a notamment été utilisé dans les années 1780 comme preuve de droits territoriaux devant les autorités britanniques nouvellement installées sur le territoire 7 . Voici le différend qui opposait à l'époque les habitants de Kanesatake et leurs voisins canadiens concernant la propriété des terres: les prêtres sulpiciens contestaient la propriété des premiers sur des champs dont ils prétendaient qu'ils leur avaient été concédés afin de pourvoir à l'instruction des populations autochtones domiciliées dans leur seigneurie du Lac-des-Deux-Montaǵnes. En 1781, afin de soutenir leur réclamation et la raison de leur requête, les chefs présentent le wampum aux autorités coloniales et, en décrivant les symboles qui y sont tissés, lui donnent la signification suivante:

Cette ligne blanche Père sur le collier décrit, tel que le veut notre coutume, la longueur de nos terres, ces figures main dans la main qui rejoignent la Croix représentent notre fidélité à notre religion que nous professons tous; le Corps représente le feu du conseil de notre Village, les deux Chiens, à chaque extrémité, doivent garder les Limites de nos Terres, et si quelqu'un perturbe notre paisible possession, ils doivent nous avertir en aboyant, et c'est ce qu'ils font depuis les trois dernières années.

(Cité dans Lainey 2013: 99)

On remarque que les motifs du collier sont explicitement décrits et expliqués dans le compte rendu de l'événement: personnages se tenant la main de chaque côté d'une croix catholique centrale, une ligne blanche sous leurs pieds et deux chiens aux extrémités (fig. 3). Les chefs agiront de la même manière et pour les mêmes raisons d'ordre territorial en 1788. L'année suivante, le wampum est officiellement présenté comme titre foncier devant le Conseil exécutif de Québec chargé de trancher. Cette preuve matérielle et les arguments avancés par les Autochtones ne firent pas le poids aux yeux des autorités coloniales qui préférèrent reconnaître les nombreux documents écrits officiels (titres, concessions, juǵement) présentés par les sulpiciens.

Comme pour le wampum wendat présenté précédemment, une fois le TDWB sorti de la communauté suite à sa vente par un particulier, son sens initial fut complètement oublié et modifié selon le bon vouloir de ses nouveaux propriétaires. Le responsable de cette vente privée est David Swan, un individu de Kanesatake qui travaillait aussi dans la région à l'est de la baie Géorǵienne en Ontario où des Kanien'kehá:ka de Kanesatake avaient formé un autre village au milieu 
du XIX ${ }^{\mathrm{e}}$ siècle (aujourd'hui Wahta Mohawks). Au mois de février 1919, dans une lettre qu'il adresse à David Ross McCord (le fondateur du musée qui porte son nom) pour accompagner le TDWB qu'il vient de lui vendre, Swan lui donne une signification qui n'a absolument rien à voir avec les discours des chefs des années 1780 :

Je vous envoie la commémoration des Ériés passant sous le règne de l'homme blanc. Des silhouettes d'hommes blancs et d'Indiens avec les mains jointes conduisant ainsi les Indiens sur les sentiers de la droiture qui est la ligne blanche sous leurs pieds. Des figures à chaque extrémité indiquant que l'Indien serait bientôt semblable à l'agneau. La croix au milieu représentant la fidélité au christianisme.

(AMM M1904, traduction de l'auteur)

Cette interprétation néglige tous les faits importants associés à l'origíne au wampum: une autre nation autochtone est désormais impliquée, les chiens de garde deviennent des agneaux soumis et toute notion de territoire est complètement abandonnée! Étant natif de Kanesatake, David Swan devait pourtant bien connaître la véritable signification de ce wampum. Était-ce une façon pour Swan d'éloigner, au moins sémantiquement, le wampum de sa communauté d'origine située seulement à une cinquantaine de kilomètres plus loin? Sachant que les chefs de la communauté auraient fortement réaǵi s'ils avaient appris la vente de l'objet, cherchait-il à brouiller les pistes?

On sait que par manque de connaissances historiques, par naïveté ou par volonté aveuǵle d'acquérir les pièces les plus prestigieuses pour son musée national en développement, McCord acceptait parfois facilement les informations accompagnant les objets qu'il achetait (McCaffrey 1992a: 110). Dans le cas de ce wampum toutefois, il fit preuve d'esprit critique et comprit qu'il était impossible qu'il soit relié aux Ériés, une nation de la région des Grands-Lacs dispersée au milieu de XVII siècle et n'ayant d'ailleurs jamais accueilli les jésuites dans ses villages. Plutôt que d'admettre que le message du wampum nouvellement acquis puisse être inconnu, McCord fabriqua une interprétation basée sur ce que David Swan, son pourvoyeur, lui avait communiqué :

Un collier très ancien et important commémorant la conversion de toute une Nation ou Tribu. Une croix au centre sur une base solide - l'idée de permanence; de chaque côté et touchant la croix un homme rouge et un homme blanc alternativement de chaque côté. Les huit participants se donnent la main pour indiquer que les Indiens sont conduits sur le chemin de la droiture, la ligne se terminant par un agneau à chaque extrémité - signifiant l'Agneau de Dieu, ou la vie paisible qu'ils ont entrepris d'adopter en se convertissant. Tout au long de la base court une ligne blanche, indiquant la nouvelle vie de paix; et à chaque extrémité sept doubles barres - l'entente.

(AMM M1904, traduction de l'auteur)

Encore une fois, comme pour le wampum des Wendat, c'est le nouveau sens, complètement erroné et fantaisiste, qui prévalut publiquement, répété ici et là dans diverses publications et ce, pour les soixantedix ans qui suivirent, c'est-à-dire jusqu'à ce que Paul Williams, un avocat et négociateur onondaǵa, publie en 1990 un article dans lequel il rattachait enfin l'objet du McCord aux documents d'archives de la fin du XVIII ${ }^{\mathrm{e}}$ siècle (Williams 1990 : 31). C'est ainsi que le sens originel du wampum, lequel affirmait la légitimité des droits fonciers des Autochtones sur une partie du territoire, a été détourné, discrédité, transformé, réapproprié.

Pendant toutes ces années, le wampum n'est jamais complètement tombé dans l'oubli pour les Kanien'kehá:ka. Depuis la fin du XvIII siècle jusqu'à aujourd'hui, ils n'ont cessé de poursuivre la lutte pour la reconnaissance de leurs droits, faisant parfois référence à ce wampum remontant à la fondation de Kanesatake, ainsi qu'aux discours de leurs chefs dans les années 1780 (Gabriel et Van den Hende 2010). Récemment toutefois, une signification passablement différente a été formulée par le Centre culturel et de langue de Kanesatake. En 2012, le musée McCord prêta le TDWB au musée national des Beaux-Arts du Québec pour l'exposition «Les arts en Nouvelle-France». Le commissaire Laurier Lacroix suivit done les procédures de l'institution et demanda l'autorisation à la communauté d'origine du wampum. Les responsables du Centre culturel et de langue de Kanesatake acceptèrent à condition que leurs voix et perspectives à propos du sens du wampum soient entendues, ce que le commissaire approuva. Reproduite dans la monoǵraphie accompaǵnant l'exposition, l'interprétation fournie par le Centre diffère substantiellement de celle présentée par les chefs en 1781 et 1788 . Nous la citons presque intégralement afin de donner toute sa place à la parole autochtone d'aujourd'hui :

Le Wampum aux deux Loups témoigne d'un accord datant de 1721 environ entre Kontinonh-seshà:ka (le peuple de la Maison Longue de Kanehsatà:ke) et les Sulpiciens, accompagnnés d'un groupe d'autochtones mohawks, nippissings et algonquins convertis au christianisme. L'entente avait pour objectif la cohabitation pacifique et sans ingérence des parties. [...]. Sur un arrière-plan de perles violettes figurent divers personnages, ainsi qu'un loup à chacune des extrémités de la ceinture. Les loups incarnent les protecteurs de la terre [...]. Les longs traits blancs représentent l'étendue du territoire. Les figures se donnent la main en guise de fidélité à leurs traditions respectives et de loyauté à l'égard du pacte de cohabitation pacifique. Le motif ap- 


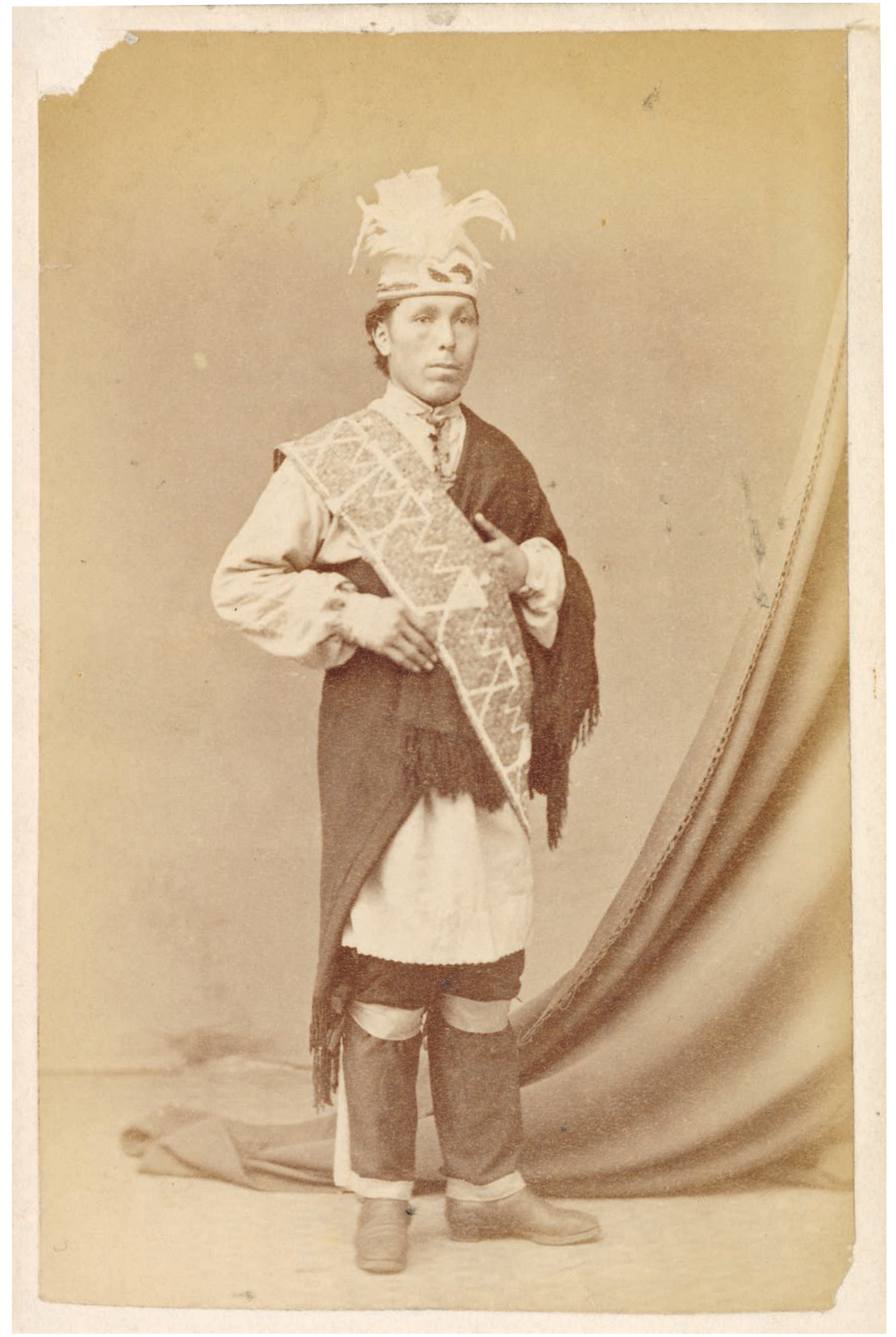

fig. 3. J. C. Parks, Le chef kanien'kehá:ka [Mohawk] Joseph Swan Onasakenrat portantle «wampum aux deux chiens» de Kanesatake, vers 1868 . Tirage sur papier albuminé, 10,2 ×6,4 cm. Collection Jean Tanguay. 


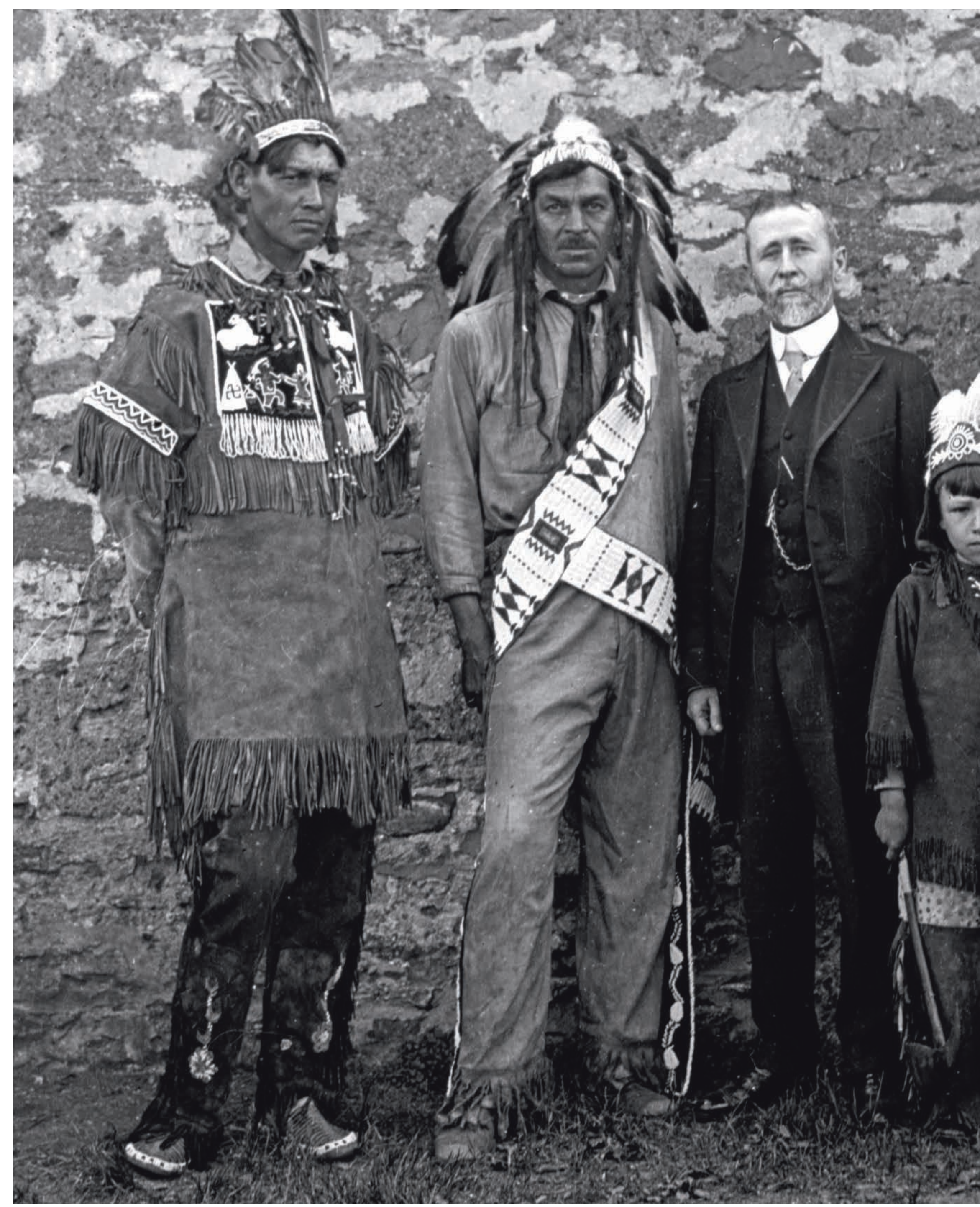




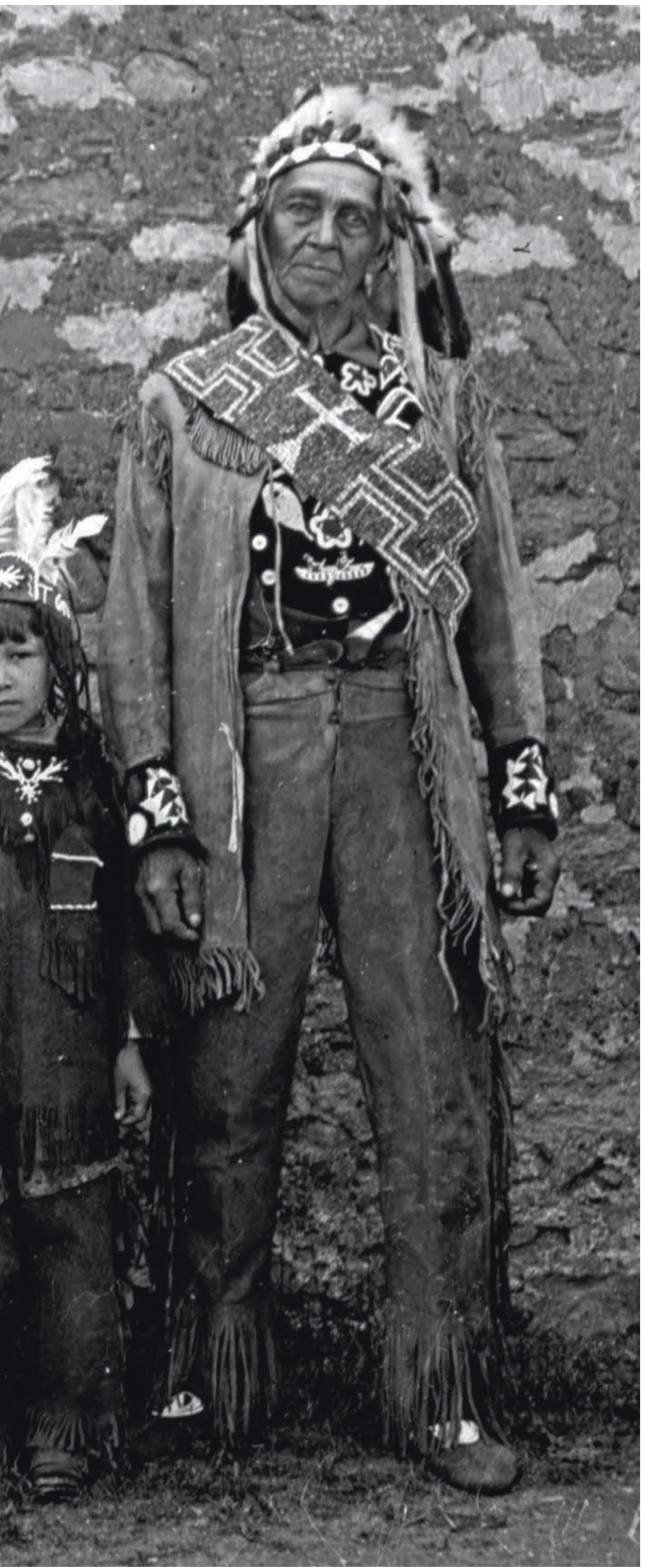

fig. 4. Edgar Gariépy

Tompagnés de Victor Morin vers 1918. Plaque de projection au gélatino-bromure d'argent, $10.1 \times 8.2 \mathrm{~cm}$ Montréal, Archives de la Ville de Montréal, BM042-Y-1-P1281, DR. Victor Morin fut un important collectionneur de la région de Montréal. L'individu à droite porte sur son épaule le wampum offert par les Wendat aux Kanien'kehá:ka vers 1677. 


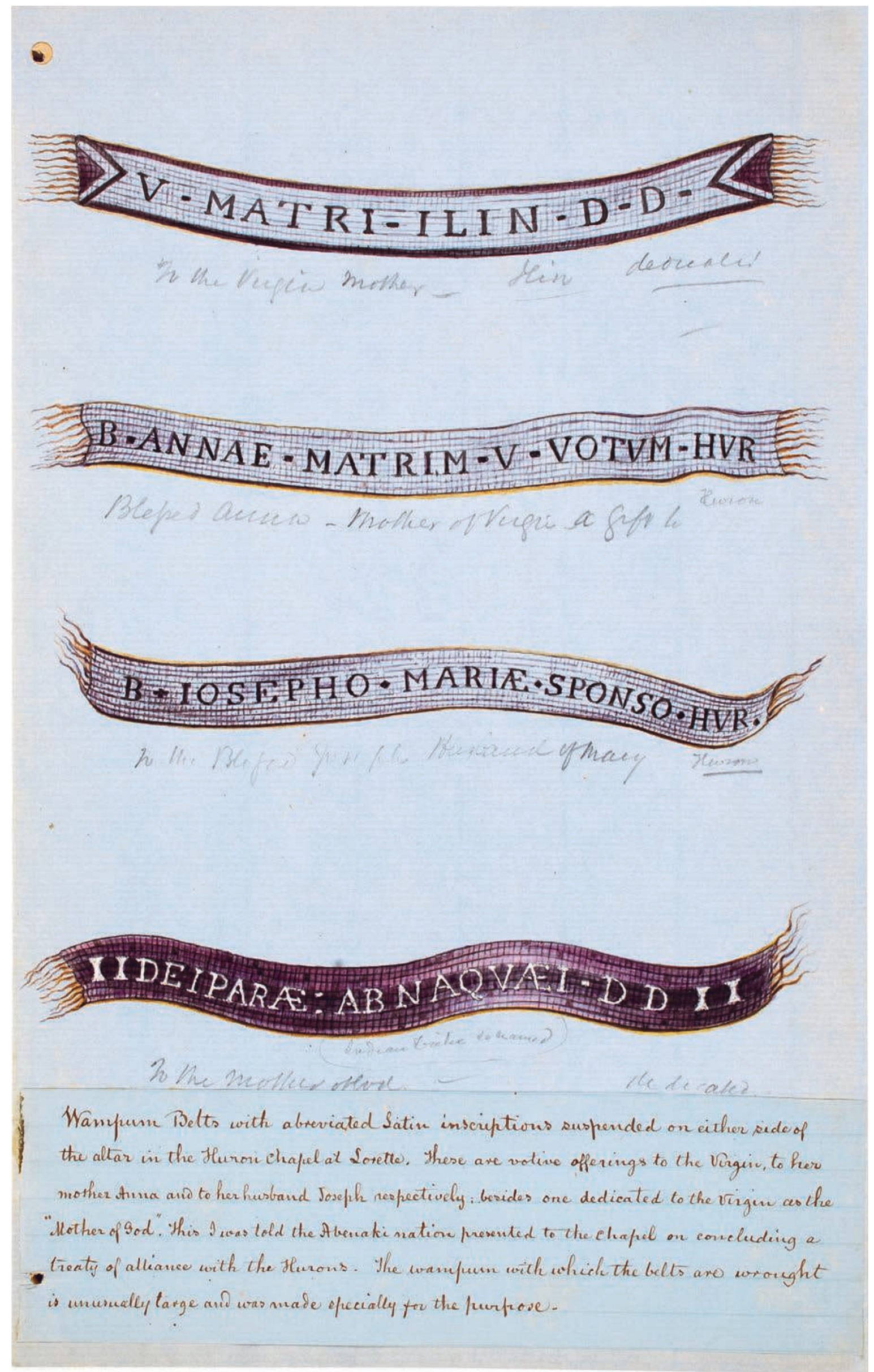

fig. 5. Samuel Douglas Smith Huyghue, Dessin des quatre colliers de wampum qui étaient suspendus dans la chapelle de Lorette [Wendake] avantl'incendie de 1862, 1846. Document graphique, dim. inconnues. Melbourne, Museums Victoria, Collection SDS Huyghue, XM 1495, Manuscript, pre-1879.

La traduction des écritures latines sur les wampums, parfois abrégées, se lit comme suit:

V'MATRI'ILIN D'D' (Don des Illinois à la Vierge Mère), B'ANNAE'MATRIM V'VOTUM'HUR (Vœu des Hurons à la Bienheureuse Anne Mère de la Vierge), B'JOSEPHO'MARIE'SPONSO'HUR' (Les Hurons au Bienheureux Joseph époux de Marie), DEIPARA'ABNAQVÆEI' D'D' (Don des Abénakis à la Mère de Dieu). 
parenté à une croix au centre de la composition correspondrait plutôt, selon l'interprétation locale, à un wampum tenu par deux personnages avec, au centre, un trait vertical symbolisant la délimitation des territoires devant être occupés et exploités. Il est peu probable qu'il s'agisse d'une croix, comme l'ont interprété initialement les anthropologues. Certaines figures sont entièrement blanches, d'autres comportent des perles violettes. Cette marque de distinction pourrait être un indice de genre, la tradition iroquoise prescrivant l'égalité des sexes. Le feu du conseil (les affaires de la nation) de Kanehsata'kehro:non est également représenté au sein des corps. Il est donc important de souligner que ce wampum constituait à la fois un symbole de cohabitation pacifique ainsi que l'affirmation, par le peuple Kanien'kehá:ka (le peuple du silex), de sa souveraineté et de son autorité sur le territoire, et non pas une concession de ses terres ni un engagement de loyauté envers les Sulpiciens. Elle affirme l'existence de Kanesatà:ke avant l'arrivée des Européens, et revendique l'appartenance du territoire aux Kanien'kehà:ka, qui devraient avoir le dernier mot sur son intendance. Malheureusement, les Sulpiciens ont d'emblée déformé le sens et l'intention première [du wampum] [...]. Les Sulpiciens prétendirent également que [le wampum] signifiait la concession par les Mohawks de Kanesatà:ke de leurs droits sur leurs terres, et par conséquent, leur allégeance. En fait, il s'agissait du contraire. Le wampum aurait dû signifier la collaboration des Sulpiciens ainsi que le respect de l'occupation et de l'exploitation des terres parles Moharwks. Le peuple de Kanesatà:ke a résisté pendant de nombreuses générations aux tentatives déployées par les Sulpiciens pour lui dérober ses terres. Quelque quatre cents ans plus tard, la lutte continue.

(Cité dans Lacroix 2012: 208)

En plus d'offrir une version contemporaine de l'histoire de la formation de leur village et d'affirmer la souveraineté ancestrale de leur peuple sur leur territoire, cette interprétation fait aussi référence aux enjeux politiques et sociaux du $\mathrm{XxI}^{\mathrm{e}}$ siècle. Sur quels éléments de la tradition orale et sur quels arguments historiques les membres de la communauté de Kanesatake se basent-ils pour fournir une interprétation qui diffère grandement des paroles de leurs propres ancêtres rapportées dans les documents d'archives, au point de les contredire sur certains points?
L'origine de cette interprétation contemporaine mériterait en soi une étude incluant des entrevues avec les gens de Kanesatake aujourd'hui.

\section{LES WAMPUMS OFFERTS EN CONTEXTE RELIGIEUX ET DEMEURÉS AU QUÉBEC}

Entre 1654 et 1716, une dizaine de colliers ont été fabriqués par des populations autochtones vivant dans des missions religieuses pour être offerts à des sanctuaires européens et canadiens. La grande majorité de ceux-ci a été produite par les Wendat et a servi d'offrandes pieuses visant à honorer la Vierge Marie et d'autres saints ainsi qu'à formuler des vœux à connotation religieuse. Certains wampums furent envoyés en France, d'autres restèrent parmi eux. Malheureusement, peu d'entre eux nous sont parvenus, celui du musée du quai Branly-Jacques Chirac et les deux exemplaires de la cathédrale de Chartres étant les seuls encore connus aujourd'hui sur la dizaine officiellement documentée dans les Relations des jésuites et dans les archives. En effet, le sort des wampums restés en Amérique n'est pas aussi heureux que celui des colliers qui traversèrent l'Atlantique il $\mathrm{y}$ a plus de trois cent cinquante ans.

Les Wendat ayant produit la majorité de ces wampums, il n'est pas étonnant que sur les murs de la chapelle de Lorette (aujourd'hui Wendake) aient été suspendus, de chaque côté de l'autel, quatre de ces wampums votifs. Lors de l'incendie qui détruisit la chapelle en 1862, on ne put malheureusement les sauver des flammes. Le seul témoignage visuel connu de ces wampums qui ponctuèrent la vie religieuse des ancêtres des Wendat se trouve à Melbourne, en Australie, dans le manuscrit d'un dénommé Samuel Douǵlas Smith Huyghue, qui, en 1846, rencontra les Wendat chez eux et prit note de l'apparence de ces quatre uniques wampums et de ce qui y était écrit (fig. 5).

Si la perte de ces inestimables wampums est dommageable à de nombreux égards, elle l'est en particulier du point de vue de la recherche proprement dite. Ce corpus de wampums très anciens aurait en effet été très utile pour aider à situer dans le temps et l'espace les différentes techniques de tissage des colliers et de perçage des perles, en servant de base comparative pour étudier et analyser d'autres wampums connus aujourd'hui. Les wampums dont le contexte de création et d'échange est précisément connu sont très rares, bien qu'il soit courant de constater, en parcourant les bases de données d'institutions muséales qui en possèdent, que les dates qu'on leur confère sont particulièrement anciennes, remontant parfois au début des années 1600. Or, on le sait, les informations qui accompagnent les wampums dans les musées ne permettent qu'exceptionnellement de les associer avec certitude à une période, ou à un groupe en particulier (Lainey 2004 et 2008). Ce type de wampums fabriqués en contexte religieux est plus facilement situable dans le temps et l'espace. Comme ils ont été fabriqués sous la supervision des jésuites, les écrits et la corres- 
8. Certains Wendate Kanien'kehà:ka d'aujourd'hui considèrent d'ailleurs qu'au-delà de l'encouragemen à la ferveur religieuse et à la fo catholique qui est rapporté dans les Relations des jésuites témoignant du don de ce témoignant du don de ce wampum, celui-ci établissait les deux villages et nations selon la géopolitique autochtone du moment laquelle motivait davantage les décisions des individus et des groupes que les intérêts strictement missionnaires. pondance de ces derniers nous permettent de savoir quand et à qui ils adressaient ces wampums. De plus, en fonction des caractéristiques matérielles qui les distinguent - ils comportent des inscriptions latines véhiculant le vœu des destinateurs -, il est relativement facile de les reconnaître et de les relier aux événements historiques documentés.

Un autre wampum généralement considéré comme un de ceux offerts en contexte religieux a été conservé et exposé pendant trois siècles dans l'église de Kahnawake. Il a été offert par les Wendat vers 1677 (fig. 4). Ce précieux wampum aurait pu sortir de la communauté, confrontée aux difficultés socioéconomiques que l'on connaît, au même moment que les deux autres présentés précédemment. En effet, des individus de la communauté ont tenté de le vendre à deux reprises, en 1923 et en 1926 . Ce wampum avait alors une valeur monétaire considérable. Le journaliste qui couvre le procès des deux hommes de 70 et 80 ans accusés de vol en 1923 souliǵne que des musées états-uniens ont offert de très fortes sommes pour se porter acquéreurs du wampum en question (The Gazette 1923). Trois ans plus tard, c'est le révérend O. Lacouture lui-même qui tente de le vendre, cette fois au musée McCord, qui avait ouvert ses portes cinq ans plus tôt. Il cherchait apparemment à en retirer une forte somme dans le but de financer des réparations au sein d'établissements religieux. Entrevoyant l'illégitimité du geste, l'assistante conservatrice du musée, Mary Dudley Muir, écrit aussitôt au ministère des Affaires indiennes pour signaler les intentions du prêtre, qui fut réprimandé par le superintendant général Duncan Campbell Scott (BAC 1923-1932).

Fait digne d'intérêt, le journaliste mentionne que la communauté de Kahnawake est alors divisée en deux factions en raison notamment des questions entourant l'élection des représentants de la nation. Ceci fait donc écho à ce qui s'est passé à Wendake à la même époque, où les chefs à vie ont été remplacés par un système électif. Certains considéraient que le wampum devait rester dans l'église pour être conservé par les prêtres, alors que d'autres jugeaient que le collier diplomatique appartenait aux «Iroquois » parce qu'il scellait une alliance avec les Wendat ${ }^{8}$.

Remarquons à nouveau que l'enjeu autour du mode de représentation politique de même que le contexte économique difficile ne sont pas étrangers au sort des wampums autrefois détenus par les chefs traditionnels. Le contexte ǵlobal du destin des wampums est le même: les structures traditionnelles se transforment et sont progressivement remplacées par des systèmes exogènes qui fonctionnent selon d'autres valeurs et philosophies. À l'échelle du Canada, les communautés autochtones sont durement touchées par les mesures économiques qui les défavorisent et par l'accès grandement restreint aux ressources du territoire. Elles sont de plus en plus marginalisées et contrôlées par l'offensive juridique et légale qui pèse sur leurs épaules, dont en particulier les clauses restrictives de la loi sur les Indiens qu'ils subissent depuis près d'un demi-siècle. Mise en application par des fonctionnaires appelés «les agents des Indiens » (les yeux et les oreilles de l'État), des policiers et des missionnaires, cette loi permet de contrôler les populations autochtones en réduisant considérablement leurs droits, en définissant et limitant leurs membres, en contrôlant parfois leurs déplacements et en décourageant ou interdisant certaines de leurs pratiques culturelles sacrées.

Dans ce contexte difficile, les communautés autochtones intéressent vivement les collectionneurs, professionnels ou amateurs, cherchant à acquérir des «reliques» de ces cultures qu'ils croient voir disparaître sous leurs yeux. Typique d'une période qualifiée d' «anthropologie de sauvegarde», cet engouement pour les objets autochtones anciens fut particulièrement marqué dans les années 1860-1920 au Canada:

Pour les anthropologues et le grand public, les groupes autochtones et leurs styles de vie distincts étaient voués à la disparition. À l'époque victorienne, collectionneurs privés et scientifiques se précipitaient pour se procurer les témoignages de ce qu'ils pensaient être les vestiges fascinants d'une race en voie d'extinction.

(McCaffrey 1992b : 34)

Cet ancien et précieux wampum a disparu depuis les années 1970. A-t-il seulement été retiré de l'éǵlise par des gens souhaitant l'extraire de son contexte religieux pour lui redonner son importance politique (comme certains tentèrent de faire dans les années 1920), ou a-t-il été vendu à un prix élevé à un particulier qui l'a ajouté à sa collection personnelle?

\section{AUJOURD'HUI : INVENTORIER ET DOGUMENTER LES WAMPUMS POUR MIEUX LES CONNAITTRE}

Les colliers de wampum sont aujourd'hui toujours aussi précieux, intrigants, convoités, entourés d'une aura de mystère et considérés comme sacrés par plusieurs individus. Preuve du maintien de leur importance historique et de leur pertinence politique et légale jusqu'à nos jours, ils sont parfois mentionnés ou présentés dans les tribunaux canadiens. En 2016, l'avocate Jacynthe Ledoux, spécialisée en droit autochtone, révélait que «depuis les années 1980, des wampums ont été invoqués devant les tribunaux canadiens dans plus d'une trentaine de causes touchant aux domaines de droit les plus variés» (Ledoux 2016: 9). Le nombre de causes ayant augmenté depuis les cinq dernières années, ce chiffre doit être revu à la hausse.

Certains font parfois, quoique très rarement, leur apparition sur des sites Internet de vente ou lors de mises aux enchères publiques. Ces pièces suscitent alors de telles réactions et questionnements qu'elles sont généralement assez vite retirées des ventes (Bruchac 2018). Depuis les années 1970, cinquante wampums ont été restitués aux Haudenosaunee et moins de dix à d'autres communautés d'origine (Stolle 2016: 256 et 305-359). Aux États-Unis, la loi NAGPRA (Native American Graves Protection and 


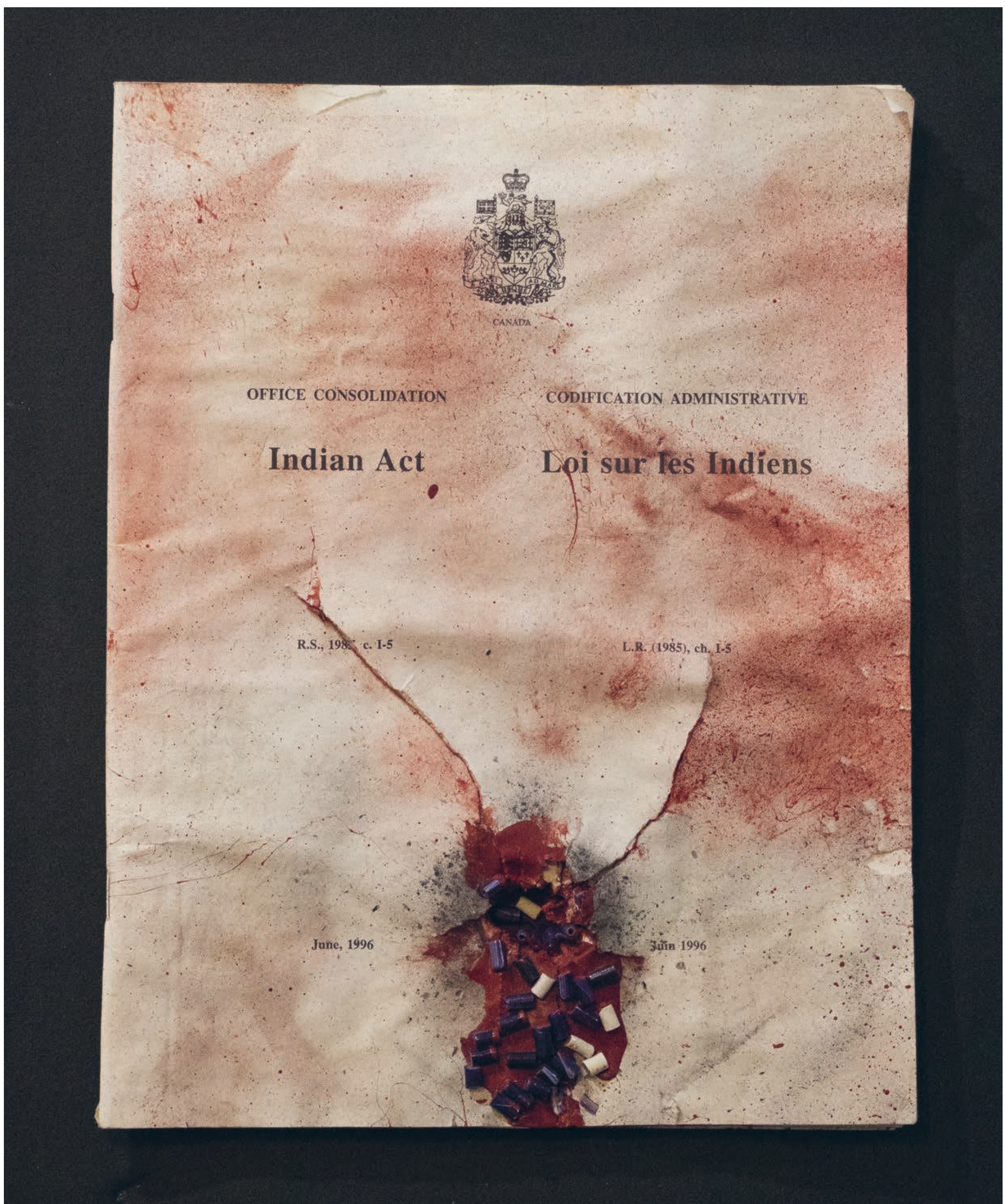

fig. 6. Teharihulen Michel Savard (artiste huron-wendat), Réciprocité, 2009. Sculpture, technique mixte, $35.5 \times 29 \mathrm{~cm}$. Avec l'aimable autorisation de l'artiste. La Loi sur les Indiens, adoptée en 1876 , donne au gouvernement canadien l'autorité exclusive de légiférer sur les «Indiens et les terres réservées pour les Indiens.»

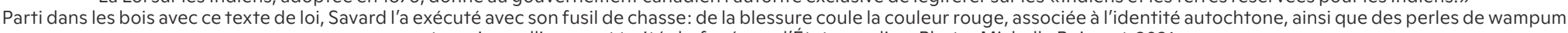
ayant servi aux alliances et traités bafoués par l'État canadien. Photo: Michelle Boisvert, 2021. 


\section{Dossier}

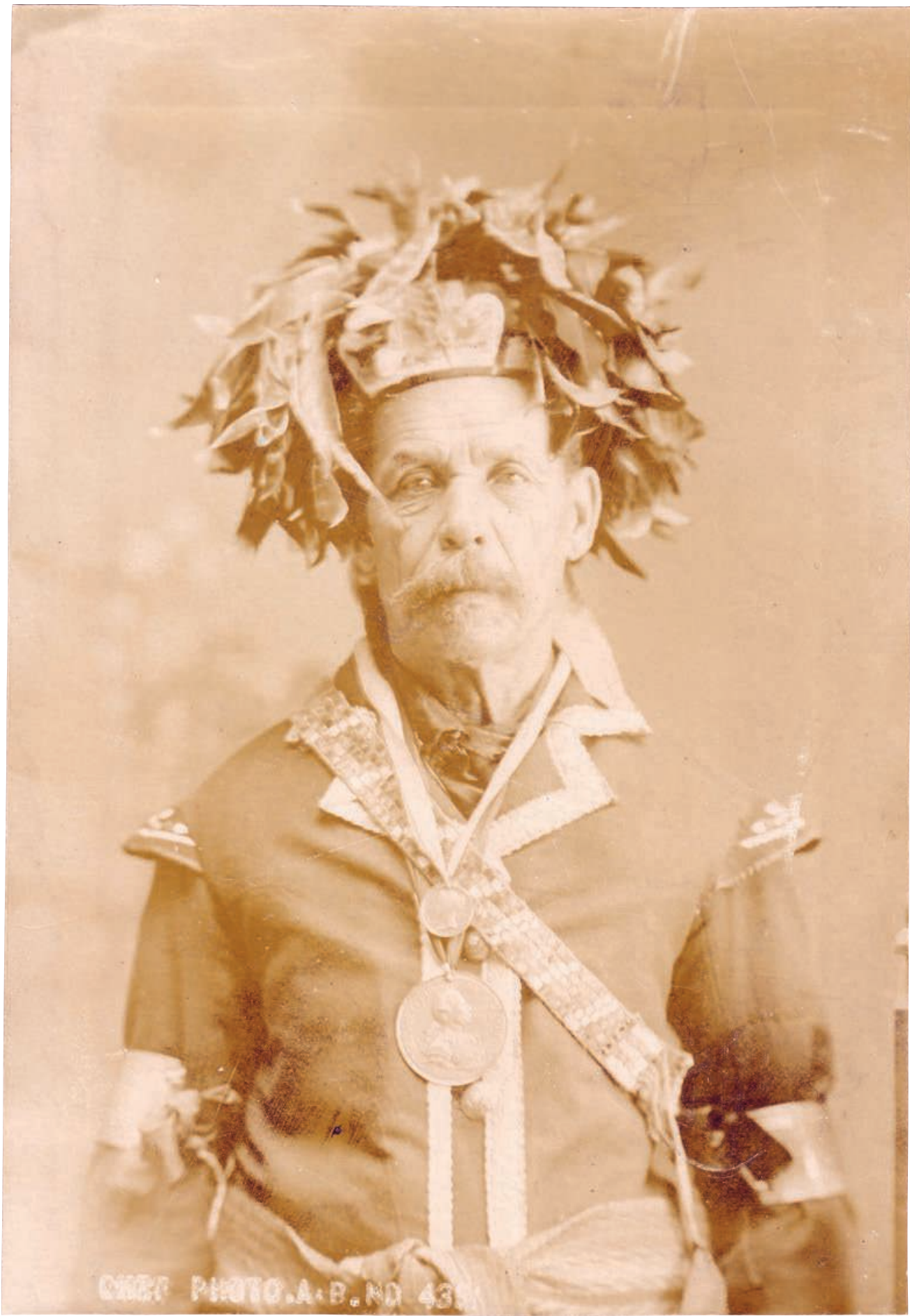

fig. 7. Alphonse Boivin, Gaspard Picard Ondiaraléthé, 1906. Épreuve à l'albumine, $15,2 \mathrm{~cm} \times 10,5 \mathrm{~cm}$.

Wendake, Québec, Archives du Conseil de la Nation huronne-wendat. Gaspard Picard Ondiaraléthé est nommé chef des guerriers selon les coutumes wendat dans les années 1870. II devient ensuite grand chef élu selon les termes de la Loi sur les Indiens de 1904 à 1909. II porte un wampum aujourd'hui conservé au Musée canadien de I'histoire ( ${ }^{\circ}$ III-H-485) de même que des médailles de George III et de la reine Victoria. 
Repatriation Act) de 1990 facilite le retour des objets culturels, des restes humains, des objets funéraires et des objets sacrés vers les communautés sources. Au Canada, bien que le Rapport du groupe de travail sur les musées et les Premières Nations de 1992 recommande aux musées de travailler en collaboration avec les peuples autochtones sur les questions de restitution (AMC/APN 1992), aucune législation nationale équivalente n'a été mise en place; c'est aux musées de se doter d'une politique concernant la restitution des objets et/ou de traiter les demandes reçues au cas par cas. Bien documenter la provenance des objets devient évidemment primordial et essentiel puisque les remettre à la mauvaise communauté reviendrait à commettre une erreur en voulant en réparer une autre (Tooker 1998). Pour ce faire, la recherche dans les archives de toutes sortes (manuscrites, iconographiques, orales), le croisement des sources et le partage des informations demeurent la voie la plus sûre.

Documenter ainsi les wampums peut donner des résultats probants. Pour que l'on puisse les reconnaître et les identifier précisément, deux conditions sont toutefois nécessaires : la description fournie dans les archives du wampum échangé doit être suffisamment exhaustive, et les motifs représentés sur le wampum doivent être suffisamment élaborés et spécifiques pour permettre de le reconnaître. De fait, les wampums comportant les motifs les plus distincts seront évidemment plus facilement reconnaissables si la description de l'objet échangé, quand elle figure dans les comptes rendus de rencontres officielles, est suffisamment précise. Le cas du TDWB en est un exemple éloquent. Par ailleurs, l'inventaire exhaustif des wampums connus à ce jour qu'est parvenu à dresser Nikolaus Stolle (2016) montre clairement que plusieurs wampums peuvent comporter des motifs très similaires, ce qui rend la tentation de les associer à leur description dans les archives toujours risquée, particulièrement quand celle-ci demeure d'ordre général comme c'est trop souvent le cas. Dans quelques rares occasions, les wampums offerts ont été dessinés par le script chargé de rapporter le contenu des discussions dans un procès-verbal (Lainey 2005: 65; Carpenter 2018).

Outre la recherche en archives, les sources iconographiques s'avèrent souvent très profitables pour reconnaître les wampums et identifier leurs propriétaires originaux. C'est peut-être plus particulièrement le cas chez les Wendat, pour qui les wampums, au moment où leur utilité diplomatique et politique s'est atténuée, devinrent des objets de parure et des symboles associés à la chefferie portés et exposés lors d'occasions spéciales. Les photographies d'époque en témoiǵnent: à partir des années 1870, les chefs sont parfois parés de colliers de wampum qu'ils portent en bandoulière, alors que les représentations plus anciennes ne montrent pas les wampums diplomatiques comme des ornements personnels (Lainey 2004: 134-136 et 150-162) [fig.7].

Enfin, il faut garder en tête que l'ensemble des wampums connus ne figurent pas tous dans les collections muséologiques à travers le monde ou au sein des communautés auxquelles ils ont été rendus. Certains, conservés dans des collections privées, reviennent parfois dans la sphère publique. Ainsi, suite à la publication en 2004 de mon livre qui porte essentiellement sur les wampums wendat, une dame de Wendake a désiré me rencontrer pour discuter d'un wampum qui est toujours en sa possession. Non seulement la propriétaire a pu en apprendre davantage sur son wampum, mais celui-ci a été exposé et mis en valeur dans le Musée huron-wendat suite à des discussions avec la famille, permettant ainsi à la nation entière de renouer avec une partie de son patrimoine.

En 2017 est survenu un événement rarissime. La photo d'un wampum a circulé au sein d'un cercle restreint de chercheurs. La famille qui le détient cherchait à retrouver les propriétaires originaux pour leur retourner ce wampum qui avait été acquis par leur père, un collectionneur de numismatique au milieu du $\mathrm{xx}^{\mathrm{e}}$ siècle. L'ayant immédiatement reconnu comme faisant partie de la collection originale de Cyrille Tessier grâce au croisement des diverses sources historiques consultées et accumulées au cours des vinǵt dernières années, j'ai pu mettre en contact la famille avec la Nation huronne-wendat afin qu'ils discutent ensemble du retour potentiel de cet important objet patrimonial. Lorsque l'on considère l'histoire parfois tumultueuse de ces objets et l'aura quasi mythique qui les entoure aujourd'hui, il faut reconnaître que la générosité du geste de la famille est absolument exceptionnelle.

Musée MeCord, Montréal, Québec jonathan.lainey@mecord-stewart.ca 


\section{Archives}

AMM (Archives du musée McCord, Montréal)

«dossier de recherche - wampum M1904».

\section{BAC (Bibliothèque} et Archive Canada) RG10, «Correspondence Regarding Theft of Wampum Belts at the Caughnawaga Agency », 1923-1932, vol. 6817, dossier 486-8-2.

\section{BAC, RG10}

«Correspondence Regardinǵ the Sale of Relics, Documents \& Antiques», 1923-1925 vol. 3231 , dossier $582-548$.

\section{BnF (Bibliothèque} nationale de France)

«Memoire concernant les Coliers de Porcelaine des Sauvages, leurs differents usages et la matière dont ils sont composés », anonyme, s. d. [vers 1725], Paris, Manuscrits occidentaux Nouvelles acquisitions françaises, vol. 2550: 24-27.

\section{Bibliographie}

AMC/APN (Association des musées canadiens/Assemblée des Premières Nations)

1992 Tourner la page: forger de nouveaux partenariats entre les musées et les Premières Nations - Rapport du Groupe de travail sur les musées et les Premières Nations, Ottawa, Ontario.

\section{Anonyme}

16 novembre 1923 «Guilty of Theft of Wampum Belt», The Gazette : 5

\section{Anonyme}

28 juillet 1898 «Old Wampum Belts - A Curious Suit Entered the New York Court»,

The Gazette : 6.

\section{Bruchac, Margaret}

2018 «Broken Chains of Custody: Possessing, Dispossessing, and Repossessing Los Wampum Belts », Proceedings of the American Philosophical Society 162 (1) : 56-105.

\section{Carpenter, Brian}

19 juillet 2018 «Manuscripts Contain Rare 1712 Wampum Drawings ", American Philosophical Society [en ligne], disponible sur: https://www.amphilsoc.org/ blog/manuscripts-containrare-1712-wampum-drawings consulté le 2 septembre 2021 .
Fenton, William $\mathbf{N}$

1989 «Return of the Eleven Wampum Belts to the Six Nations Iroquois Confederacy on Grand River, Canada », Ethnohistory 36: 392-410.

Gabriel, Brenda et Van den Hende, Arlette Kawanatatie 2010 À l'orée des bois une anthologie de l'histoire du peuple de Kanehsatà:ke. Centre culturel et de lanóue

Tsi Ronterihwanónhnha ne Kanien'kéha, Kanehsatake.

Hale, Horatio E.

1897 «Four Huron Wampum Records: A Study of Aborigina American History and

Mnemonic Symbols », Journal of the Anthropological Institute of Great Britain and Ireland 26: 221-247.

\section{Jones, Peter}

(Kahkewaquonaby)

1861 History of the Ojebrway Indians: With Especial

Reference to Their Conversion to Christianity. Londres, A.W. Bennett.

\section{Lacroix, Laurier}

2012 Les Arts en Nouvelle

France. Québec, musée national des Beaux-Arts du Québec/ Publications du Québec.

\section{Lainey, Jonathan} et Whitelaw, Anne

2021 «The Wampum and the Print: Objects Tied to Nicolas Vincent Tsawenhohi's London Visit, 1824-1825», in Beverly Lemire, Laura Peers, et Anne Whitelaw (dir.), Object Lives and Global Histories in Northern North America: Material Culture in Motion, c. 1780-1980. Montréal, MQUP: 176-202.

\section{Lainey, Jonathan}

2004 La «monnaie des Sauvages»: les colliers de wampum d'hier à aujourd'hui. Québec, Septentrion.

2005 «Les colliers de porcelaine de l'époque coloniale à aujourd'hui », Recherches amérindiennes au Québec 35 (2) : 61-73

2008 «Le prétendu wampum offert à Champlain et l'interprétation des objets muséifiés Revue d'histoire de l'Amérique française 61 (3-4) : 397-424.

2013 «Les colliers de wampum comme support mémoriel: le cas du Two-Dog Wampum», in Alain Beaulieu, Martin Papillon et Stephan Gervais, Les Autochtones et le Québec: des premiers contacts au Plan Nord. Montréal, Presses de l'Université de Montréal : 93-111

\section{Ledoux, Jacynthe}

2016 « Sur les traces des wampums devant les tribunaux canadiens : réflexions sur l'état du dialoǵue internormatif entre traditions juridiques autochtones et étatique», mémoire de maîtrise. Montréal, faculté de droit, université McGill.
Lindsay, Lionel Saint-George

1900 Notre-Dame de la JeuneLorette en la Nouvelle-France. Montréal, La Cie de Publication de la Revue canadienne.

\section{McCaffrey, Moira T.}

1992a «Rononshonni le Bâtisseur: la collection McCord d'objets ethnographiques », in Pamela Miller et al. La Famille McCord: une vision passionné The McCord Family, A Passionate McCord Family, A Passionat
Vision. Montréal, musée MeCord d'histoire canadienne 102-114

1992b Aux couleurs de la terre: héritage culturel des Premières nations/Wrapped in the Colours of the Earth: Cultural heritage of the First Nations. Montréal, musée McCord d'histoire canadienne.

\section{Richardson,}

Edgar Preston (dir.)

1951 The French in America 1520-1880: An Exhibition

Organized by the Detroit

Institute of Arts to Commemo-

rate the Founding of Detroit

by Antoine de Lamothe

Cadillac in the Year 1701

Detroit, Detroit Institute of Arts.

Stolle, Nikolaus

2016 Talking Beads: The History of Wampum as a Value and Knowledoe Bearer From Its Very First Beginnings Until Today. Hambourg, Dr. Kovac.

\section{Tooker, Elisabeth}

1998 «A Note on the Return of Eleven Wampum Belts to the Six Nations Iroquois Confederacy on Grand River, Canada», Ethnohistory 45 (2). 219-236.

Williams, Paul

1990 «Reading Wampum Belts as Living Symbols », Northeast Indian Quarterly 7 (1) : 31-35.
Ci-contre

J. C. Parks, Le chef kanien'kehá:ka [Mohawk] Joseph Swan Onasakenrat portantle "wampum aux deux chiens») de Kanesatake vers 1868. Tirage surpapie lbuminé, $10,2 \times 6,4 \mathrm{~cm}$. Collection Jean Tanguay.

ouverture

Alphonse Boivin, Gaspard Picard Ondiaraléthé, 1906. Épreuve à l'albumine, $15,2 \mathrm{~cm} \times 10,5 \mathrm{~cm}$. Wendake, Québec, Archives du Conseil de la Nation huronnewendat. Gaspard Picard

Ondiaraléthé est nommé chef des guerriers selon les coutumes gueriers selon les coutumes II devient ensuite grand chef élu selon les termes de la Loi sur les Indiens de 1904 à 1909. II porte un wampum aujourd'hui conserve au Musée canadien de l'histoire médailles de George III et de la reine Victoria (détail). 


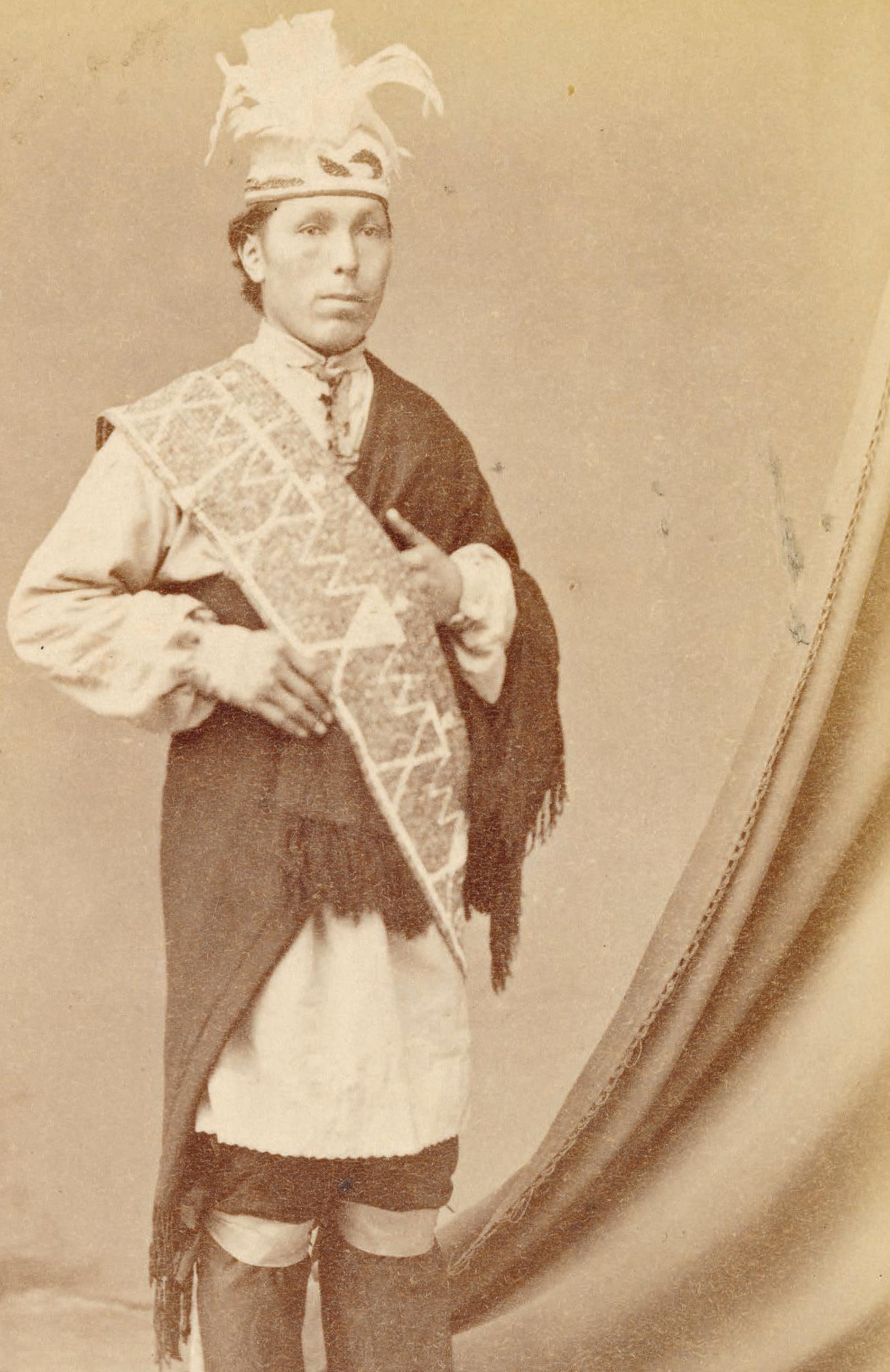

